\title{
NHS trust stands by doctors who set up clinic for private work
}

Worcestershire Acute Hospitals Trust would like to emphasise that it contracts services from Spire not Worcestershire Bowel Clinic. As stated in the News story (BMJ 2016;354:i4157, doi:10.1136/bmj.i4157), Worcestershire Bowel Clinic is based at the private Spire South Bank Hospital in Worcester. 